\title{
Contribution à la connaissance des hôtes intermédiaires et à l'étude du cycle évolutif de Paramphistomum cervi (Schrank, 1790), (Trematoda, Paramphistomidæ), à Madagascar
}

\author{
par S. GRETILLAT
}

Dans le cadre des recherches attribuées au service de parasitologie du Laboratoire Central de l'Elevage de Tananarive, figure l'étude des helminthiases des jeunes bovidés à Madagascar.

Ces parasitoses dont l'étiologie est variée, surtout dans les régions de basse altitude à climat chaud et humide, font payer tous les ans un lourd tribut à l'élevage malgache en provoquant en fin de saison sèche, une forte mortalité parmi les jeunes animaux.

Parmi ces helminthiases, l'une d'elles, la gastrothylose, a déjà fait l'objet d'une note préliminaire (Grétillat, 1957), et l'étude du cycle de son agent pathogène, Carmyerius dollfusi Golvan, Chabaud et Grétillat, 1957, a été entreprise.

C'est au cours des recherches sur le cycle expérimental en laboratoire de ce trématode, et grâce aux résultats des enquêtes réalisées sur le terrain pour cette helminthiase, que nous avons été amené à étudier et à rechercher quels pouvaient être, à Madagascar, les mollusques hôtes intermédiaires d'un trématode très souvent associé à $C$. dollfusi, à savoir le banal Paramphistomum cervi (Schrank, 1790).

Nous n'entrerons pas dans la discussion de savoir si $P$. cervi est pathogène pour son hôte ou s'il est seulement un simple commensal comme le prétendent beaucoup d'auteurs. Cela déborderait le cadre de cette note. Nous voudrions seulement dire que le travail que nous exposons ici a été réalisé afin de mettre un peu d'ordre dans les résultats des observations que nous avons pu recueillir au cours des enquêtes parasitologiques faites sur le terrain.
Il est en effet assez déconcertant pour celui qui dissèque des mollusques d'eau douce provenant des régions comme, par exemple, celles de Marovoay-Majunga (Côte Ouest) ou de Brickaville (Côte Est), de trouver dans un seul gite à mollusques, qu'il soit permanent ou récent, des bulins et des planorbes de différentes espèces dont les tissus, l'hépato-pancréas, le pied, sont envahis par des formes larvaires pouvant être rapportées à plusieurs espèces de trématodes. Furcocercaires, xiphidocercaires, cercaires de paramphistomidés, de forme, de structure et de dimensions variées créent une confusion qui n'est pas pour faciliter le travail.

Parmi les formes larvaires pouvant être rapportées à des Paramphistomidae, nous trouvons, chez les mollusques d'eau douce à Madagascar, cinq formes qui, tout en présentant des caractères communs (cercaires très pigmentées, avec deux ventouses l'une antérieure, l'autre postérieure, présence de deux taches oculaires, queue simple, enkystement rapide, etc.), n'en sont pas moins très différentes dans leurs détails de structure.

Afin de procéder par élimination, nous avons tout d'abord entrepris de réaliser au laboratoire le cycle expérimental de $P$. cervi.

Le premier travail sur le cycle évolutif de ce trématode date de 1896 et a été fait par Loos qui travaillait dans les régions de la Basse-Egypte. Cet auteur disait déjà :

"De nos jours, ce n'est plus l'organisation seule d'une forme qui représente le but de l'étude scientifique, mais son cycle vital, ses relations avec des formes voisines et avec toute la série 
animale. "Cet auteur faisait ainsi le point sur limportance de la connaissance exacte du cycle biologique d'un parasite tant au point de vue nosologique qu'épidémiologique. Le travail de Loos confirmait l'hypothèse que Sonsino avait émise en 1892, à savoir que la Cercaria pigmentata de cet auteur était bien la cercaire de $P$. cervi.

Plus tard, Takahasi en 1928, étudie le cycle de ce paramphistome, cependant que Brumpt en 1929 et 1936 donne quelques précisions en ce qui concerne l'hôte intermédiaire de $P$. cervi en Corse ainsi que les résultats des observations qu'il a pu faire au cours de dissections de Bulinus contortus infestés naturellement.

Dinnick (1951) travaillant sur les cycles des Paramphistomidae existant au Kenya, trouve dans cette région deux hôtes intermédiaires pour ce trématode, mais dans une note personnelle, cet auteur nous signale qu'il ne s'agit pas de $P$. cervi mais de Paramphistomum microbothrium Fischœder, 1901.

A Madagascar, aucun cycle de trématode n'a encore été décrit ou déterminé, et à notre connaissance les seules enquêtes faites sur le terrain au sujet des formes larvaires de trématodes, sont celles de Grjebine et Menache en 1953, en région d'Ambositra, et la même année celle que $\mathrm{Grjebine} \mathrm{a} \mathrm{faite} \mathrm{en} \mathrm{région} \mathrm{de} \mathrm{Marovoay.}$

$\mathrm{Au}$ cours de ces enquêtes malacologiques et épidémiologiques entreprises afin de mettre sur pied des méthodes de lutte contre l'endémie bilharzienne de ces régions, les différentes espèces de mollusques d'eau douce récoltées et disséquées par les auteurs s'étaient montrées infestées par des furcocercaires et par des formes larvaires qu'ils rapportèrent à Paramphistomum sp., voire à $P$. cervi.

Seule l'étude du cycle expérimental faite au laboratoire pouvait donner une solution au problème de savoir quels étaient le ou les hôtes intermédiaires de $P$. cervi à Madagascar.

Les résultats que nous donnons ici sont ceux de recherches poursuivies durant environ une année.

En avril 1957, une enquête malacologique faite en région de Vohémar (côte Nord-Est) avec Grjebine $\left({ }^{*}\right)$, entomologiste à l'Institut de la Recherche Scientifique à Madagascar, nous montre que beaucoup d'Anisus crassilabrum

(*) Nous tenons à remercier ici notre camarade et ami Grjebine, de l'Institut de la recherche' scientifique à Madagascar, pour toute l'aide qu'il nous a fournie en ce qui concerne l'élevage des mollusques d'eau douce au laboratoire.
(Morelet) de cette région sont infestés par des formes larvaires de Paramphistomidae.

Plus tard, en juillet de la même année, nous faisons la même constatation en région de la Sakay (ouest de Tananarive) sans pouvoir pour cela attribuer les cercaires qui sortent de ces mollusques à l'espèce $P$. cervi.

C'est ainsi qu'en octobre 1957, nous décidons de mettre en élevage au laboratoire les cinq espèces de mollusques d'eau douce trouvées le plus couramment dans les rizières malgaches.

Pour réaliser en effet un cycle expérimental de trématode au laboratoire, il est indispensable d'avoir à sa disposition, et en quantité suffisante, des mollusques non infestés. Le seul moyen pratique de se procurer un tel matériel est d'avoir recours à l'élevage.

Les espèces suivantes ont été mises en élevage :

Bulinus liratus (Tristram) souche provenant du parc zoologique de Tananarive.

Bulinus mariei (Crosse) souche provenant de Miadana-Majunga (Centre de Recherches Zootechniques de la Zone Sédimentaire).

Biomphalaria madagascariensis Smith, souche provenant de la région B.D.P.A. Sakay.

Anisus crassilabrum (Morelet) souche provenant de Vohémar.

Limnaea hovarum (Tristram) souche provenant de la Mandraka (P. km 67 de la route TananariveTamatave).

Nos premiers essais d'élevage furent assez décevants, surtout pour l'espèce $B$. mariei qui ne se reproduit que très lentement en aquarium à la température ambiante qui est parfois assez basse à Tananarive.

Les essais d'élevage faits en chauffant l'eau des aquariums à l'aide de chauffe-eau utilisés pour les élevages de poissons d'agrément, ne donnent que des résultats médiocres en raison de la difficulté de maintenir une température à peu près constante.

Nous avons obtenu de bons résultats en disposant les aquariums dans un petit laboratoire chauffé à $28^{\circ}-30^{\circ}$. L'oxygénation des aquariums étant couplée au circuit électrique de chauffage, on obtient ainsi un automatisme à peu près complet ne nécessitant qu'un minimum de surveillance.

En ce qui concerne la flore et le sol des aquariums, la fréquence de leur vidange, l'alimentation des mollusques, les cadences de reproduc- 


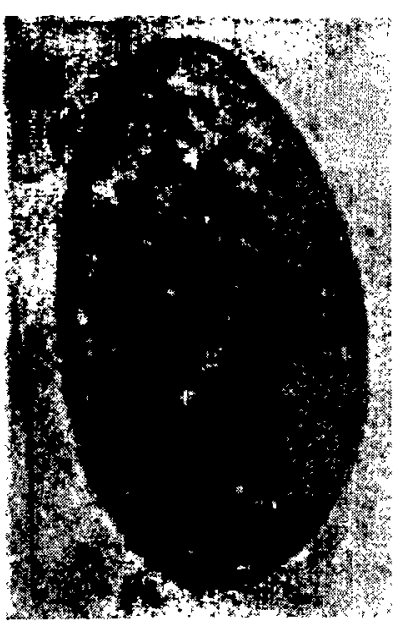

Fig. 1. - Euf de P. cervi au moment de la ponte.

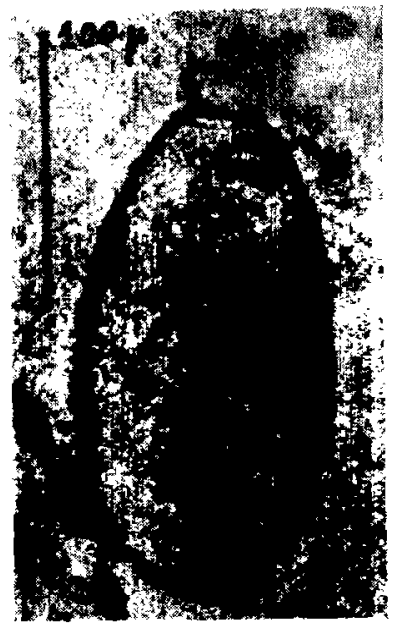

Fig. 4. - Euf éclos.

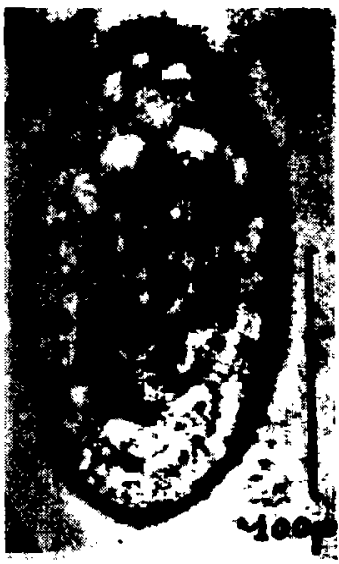

Fig. 2. - Euf au bout de 6 jours d'incubation.

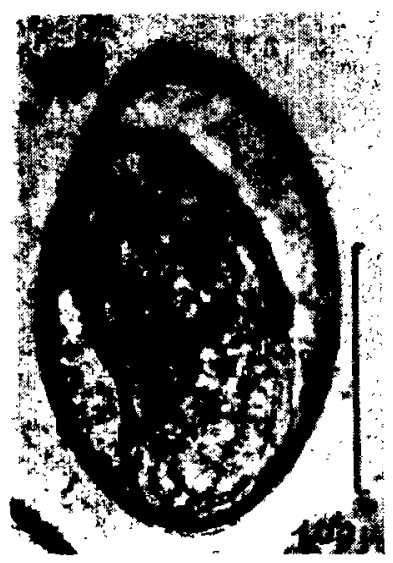

Fi. 3. - Euf au bout de 9 jours d'incubation.

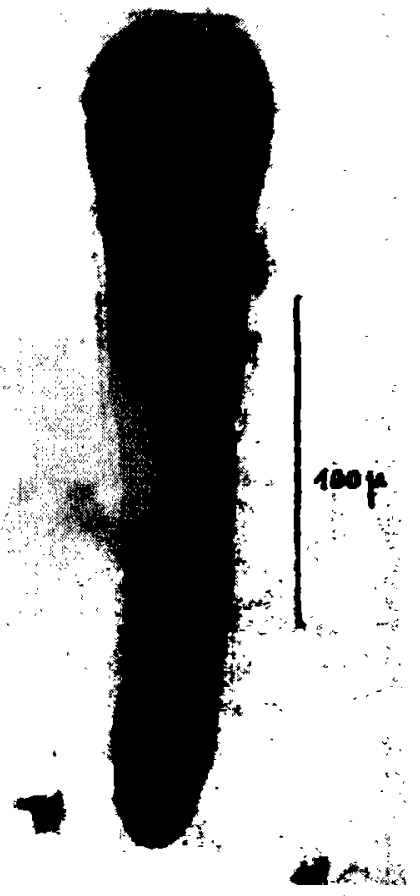

Fig. 5. - Miracidium après coloration vitale. 


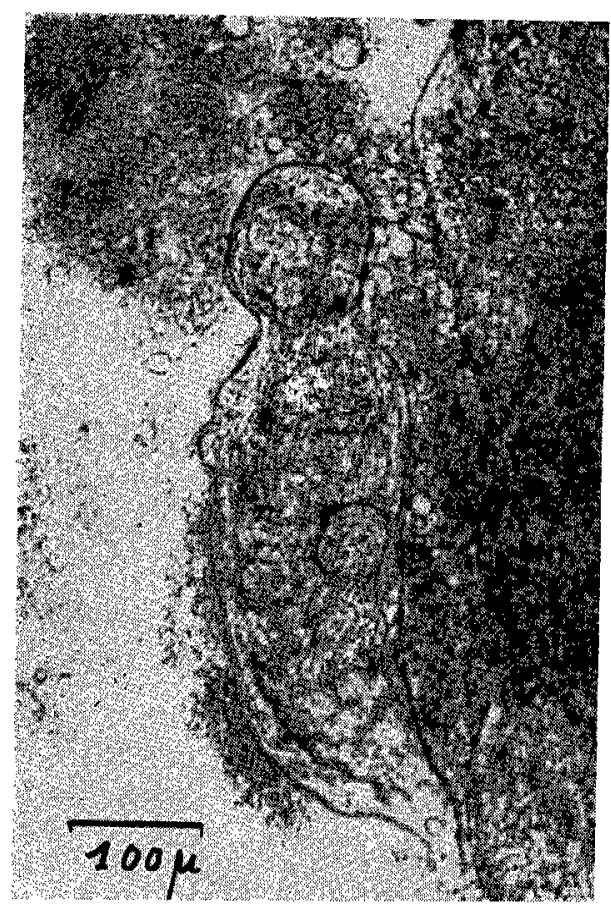

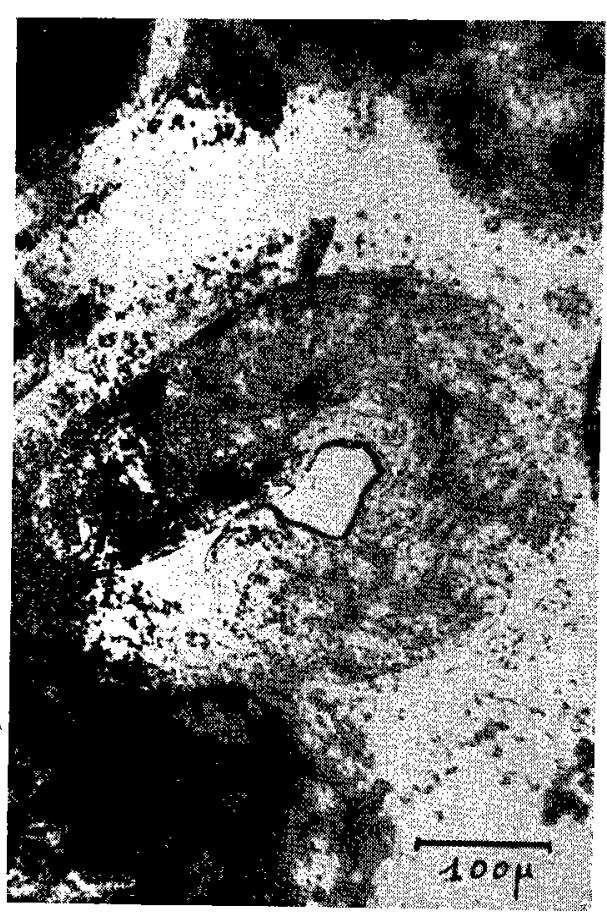

Fig. 7. - Sporocystes à différents stades de développement.

Fig. 6. - Sporocystes à différents stades de développement.

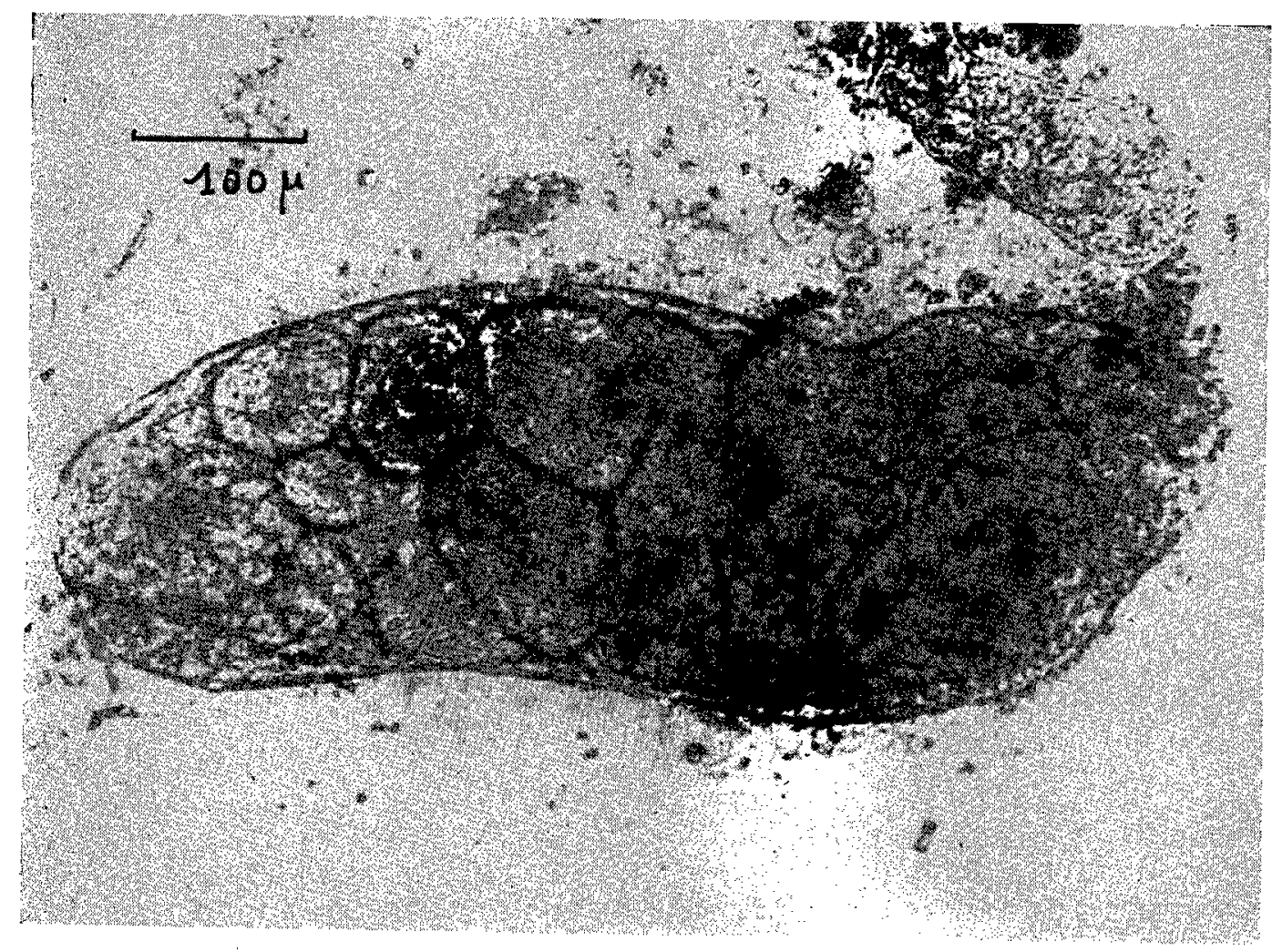

Fig. 8. - Sporocystes à différents stades de déve'oppement. 
tion de ces derniers, etc. toutes choses très importantes pour réussir de beaux élevages, elles seraient trop longues à exposer ici et déborderajent du cadre de cette communication. Nous les passerons sous silence.

\section{CYCLE EXPÉRIMENTAL}

Pour faciliter le travail et ne pas être obligé de trier dans les excréments de bovidés les cufs de $P$. cervi mélangés à ceux de $C$. dollfusi et souvent à ceux de Paramphistomum botriophoron (Braun, 1892) Fischoeder, 1901, nous n'avons pas suivi la technique de Loos, mais utilisé la méthode qui consiste à obtenir des œufs à partir de pontes artificielles.

Les prélèvements sont faits dans les panses de bovins abattus aux abattoirs municipaux de Tananarive. Ces animaux proviennent de différentes régions de l'Ile, et il est ainsi possible de se procurer facilement des trématodes des différentes espèces parasites du rumen des bovidés.

Les parasites sont amenés au laboratoire où ils sont triés par espèces, puis lavés à l'eau physiologique pour enlever de leur cuticule le maximum d'impuretés qui s'y trouvent fixées. Ils sont ensuite mis à pondre dans de l'eau physiologique à 9 p. 1.000 (technique utilisée par TimonDavid et par Dinnick). Les pondoirs sont laissés à l'étuve 24 heures à $37^{\circ}$, puis les pontes sont dénombrées et leur qualité contrôlée.

La valeur des pontes varie suivant l'âge des helminthes et on essaye d'obtenir des lots d'œufs présentant une plaque germinative bien nette et bien développée.

Les cufs sont lavés à l'eau de rizière filtrée, puis mis à incuber dans le même milieu à la température de $28^{\circ}-30^{\circ}$.

\section{Incubation de l'ceuf}

A intervalles réguliers, des prélèvements sont opérés dans les incuhateurs afin de suivre l'évolution du massif cellulaire interne de l'cuf, vérifier le temps d'incubation et la date des éclosions qui, en général, s'étalent sur plusieurs jours, les oufs étant pondus à différents stades d'évolution.

Cuf au moment ce la ponte :

Longueur : 150 à 180 ; largeur : 70 à $80 ;$.

Pourvu d'un opercule nettement visible, l'œuf présente une double enveloppe avec à son inté- rieur un massif interne plus ou moins différencié où on distingue des amas cellulaires représentant la masse vitelline. Au centre, plus ou moins apparentes, 3 à 5 cellules constituent la " plaque germinative ", point de départ du futur embryon.

Après 6 à 7 jours d'incubation, cette plaque s'est développée au point d'occuper la moitié du volume de l'cuf, cependant que les masses vitellines ont peu à peu disparu pour laisser la place à des vacuoles placées tout contre la coque de l'cuf.

Le $9^{e}$ ou $10^{e}$ jour, l'embryon s'est considérablement développé et occupe les trois quarts de l'cuf ; il s'agite en tous sens, mais ses mouvements dans le sens antéro-postérieur sont les plus marqués. Il est coincé entre une grande vacuole et la paroi de l'œuf. On peut distinguer déjà quelques détails de son organisation interne. L'ouf éclot lorsque l'incubation a lieu à $28^{\circ}-30^{\circ}$, entre les $11^{\mathrm{e}}$ et $14^{\mathrm{e}}$ jours. Le miracidium sort activement de l'œuf en faisant sauter l'opercule.

\section{Miracidium}

Très mobile à sı sortie de l'œuf, il se déplace rapidement à la recherche d'un mollusque hôte intermédiaire. Entre lame et lamelle et à $28^{\circ}$, nous avons observé une durée de survie de 12 à 24 heures. Les mouvements qui sont très rćpides dans les premières heures diminuent d'intensité vers la sixième heure pour devenir de plus en plus lents. L'attraction miracidienne sem le diminuer au fur et à mesure que le miracid.um vieillit.

Après coloration vitale, les cils qui le recouvrent apparaissent très nettement et il est même possible de distinguer les 20 cellules en plaques disposées en 5 rangées qui constituent la cuticule.

Nous trouvons pour le miracidium de $P$. cervi les dimensions suivantes : longueur : 200 à $225 \%$; largeur : 65 à $70 \%$ (variables suivant le degré d'élongation).

En ce qui concerne son organisation interne, elle apparait extrêmement complexe. On trouve, en avant des deux entonnoirs ciliés décrits par Loos, un massif cellulaire annulaire que cet auteur prétend être le système nerveux. La partie moyenne du miracidium est occupée par un amas important de cellules arrondies alors que dans la partie subterminale des éléments semblables au nombre de 15 à 20 sont disposés suivant des rangées longitudinales. 


\section{Infestation_des"mollusques :}

La plupart des auteurs ayant travaillé sur les cycles expérimentaux de trématodes sont $d$ 'accord pour admettre que l'infestation des mollusques à partir des miracidia est le temps le plus délicat et celui qui réserve le plus de déconvenues. des mollusques. Ce n'est que par tâtonnements successifs et par l'évaluation approximative du " taux d'agressivité " des miracidia qu'on arrive à avoir un nombre suffisant de mollusques infestés.

Beaucoup d'individus meurent aussi simple-

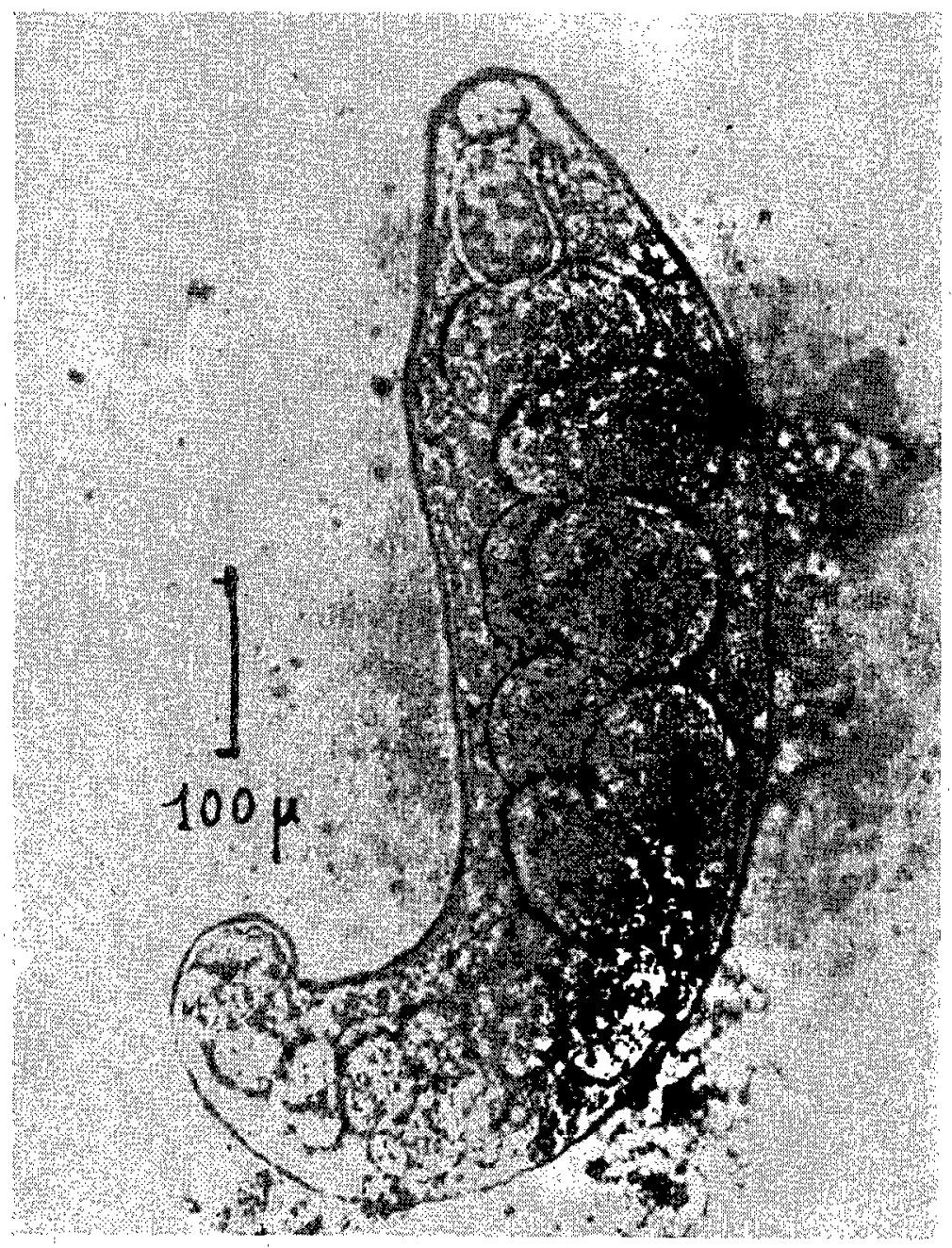

Fig. 9. - Rédie contenant de jeunes cercaires.

Le principe consiste à mettre en présence des mollusques à infester une certaine quantité de miracidia pendant un temps nécessaire à leur infestation.

Dans le cas où le nombre de miracidia n'est pas assez important pour celui des mollusques, ces derniers ne sinfestent pas; dans le cas contraire, il y a infestation massive et on aboutit dans les jours qui suivent à une mortalité élevée ment à cause des manipulations successives auxquelles on les soumet durant l'opération.

N'ayant aucun renseignement sur le mollusque hôte intermédiaire de $P$. cervi à Madagascar, nous avons été obligé de faire des essais d'infestation sur les cinq espèces trouvées le plus couramment dans les rizières malgaches.

Une première expérience est tentée avec 25 Anisus crassilabrum, 25 Biomphalaria mada- 
gascariensis, 24 Limnaea hovarum et 30 Bulinus mariei. Nous observons dans la semaine qui suit l'infestation une mortalité élevée dans toutes les espèces. Les dissections commencées vers le $8^{\mathrm{e}}$ jour et terminées le $20^{\mathrm{e}}$ ne donnent aucun résultat.

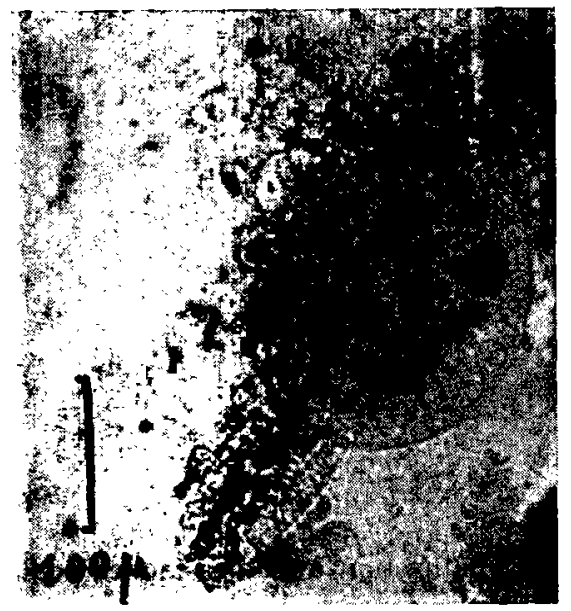

Fig. 10 - Jeunes cercaires dans les tissus sous-tégumentaires.
Nous recommençons l'expérience avec 20 spécimens de chacune de ces espèces (temps d'infestation 4 heures).

Le taux de mortalité n'est que de 20 p. 100.

Deux mollusques de chaque espèce sont disséqués les $8^{\mathrm{e}}, 10^{\mathrm{e}}$ et $12^{\mathrm{e}}$ jours après l'infestation et ne donnent aucun résultat positif.

Le $13^{\mathrm{e}}$ jour, nous trouvons un $B$. mariei présentant dans sa cavité viscérale 7 sporocystes dont 3 bien développés avec une ventouse nettement distincte. Le 16e jour, dans un mollusque de la même espèce, nous trouvons dans l'hépato-pancréas 3 sporocystes adultes et 8 rédies.

Le $20^{\mathrm{e}}$ jour, un Bulinus mariei est trouvé infesté par 5 sporocystes âgés, 18 rédies adultes et 6 jeunes rédies filles. Les tissus situés immédiatement sous le tégument présentent 22 cercaires jeunes de faibles dimensions mais possédant déjà leurs deux taches oculaires nettement arrondies.

Nous attendons le $30^{\mathrm{e}}$ jour pour faire d'autres dissections qui nous donnent $2 B$. mariei infestés massivement avec une cinquantaine de rédies mères, filles et petites-filles ayant produit des cercaires plus ou moins jeunes en voie de maturation sous le tégument du mollusque et de nombreuses cercaires mûres sur le point de sortir.

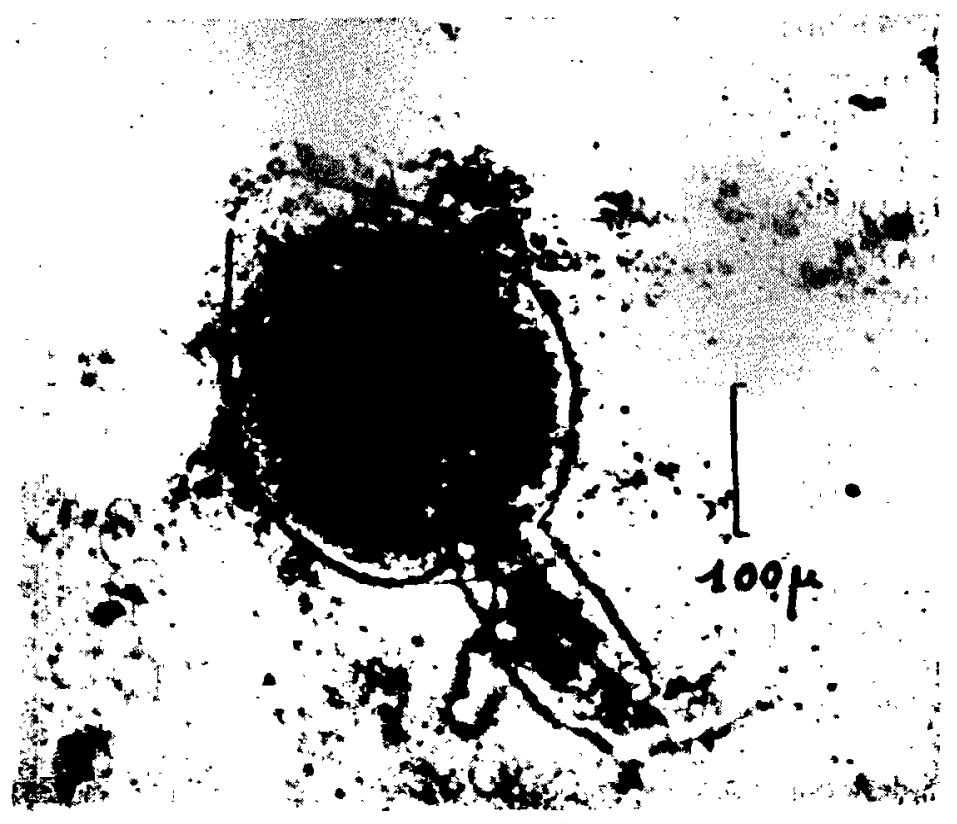

Fig. 11. - Jeunes cercaires dans les tissus sous-tégumentaires. 
Dans cette expérience, compte tenu de la mortalité ayant suivi l'infestation, un tiers des $B$. mariei a pu être infesté. Aucun spécimen des autres espèces n'a pu l'être. filles et petites-filles). Dans les tissus situés à la périphérie de l'hépatopancréas et juste au-dessous du tégument du mollusque, nous mettons en évidence une centaine de jeunes cercaires

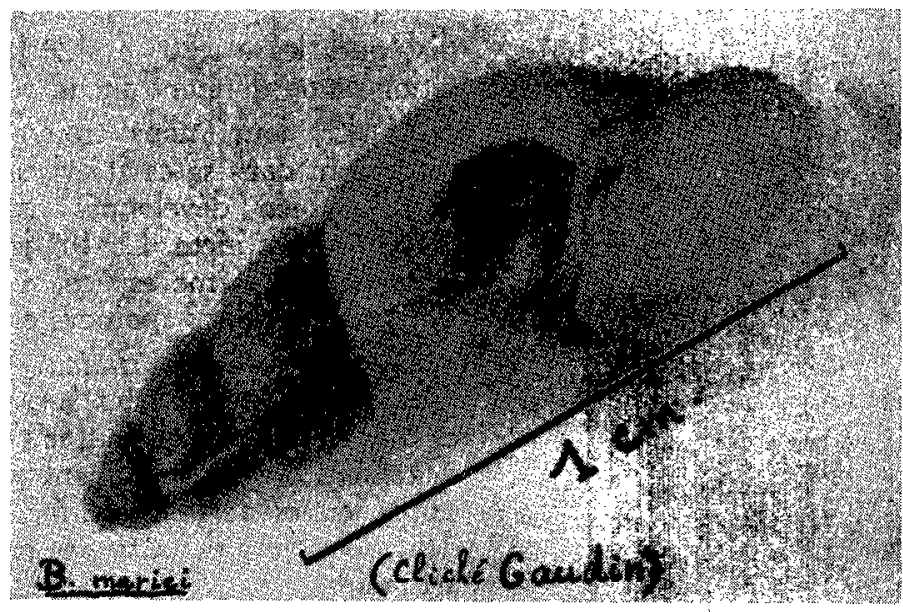

Fig. 12. - B. mariei.

Au cours d'un deuxième essai d'infestation, nous avons ajouté aux espèces précédentes le $B$. liratus.

L'expérience a été faite avec 20 Anisus crassilabrum, 25 Limnaea hovarum, 22 Biomphalaria madagascariensis, 15 Bulinus mariei et 20 Bulinus liratus.

Durée d'infestation : 4 heures.

Nous enregistrons une mortalité élevée parmi les $B$. liratus dans les 8 jours suivants.

Nous commençons les dissections 13 jours après l'infestation des mollusques.

Nous trouvons dans la cavité viscérale d'un B. mariei, 9 sporocystes de 7 à $900 \mu$ de longueur dans lesquels nous remarquons de jeunes rédies en formation, sur le point de sortir du sporocyste.

Dans l'hépatopancréas du même mollusque nous trouvons 10 jeunes rédies.

A la dissection d'un $B$. liratus, nous n'isolons que 5 très jeunes sporocystes animés de mouvements extrêmement lents et présentant chacun des massifs cellulaires en voie de différenciation sans que nous puissions distinguer des rédies nettement organisées.

Le 20e jour après l'infestation, nous disséquons un $B$. mariei dont l'hépatopancréas est bourré de rédies de différentes tailles (rédies mères, en voie de maturation avec des taches oculaires plus ou moins développées et un corps clair. On distingue les deux trajets sinueux des canaux excréteurs. Quelques-unes de ces cercaires sont déjà à un stade plus avancé et la pigmentation de leur tête leur donne une coloration plus foncée.

Le même jour, nous disséquons $2 B$. liratus et isolons dans leur hépatopancréas 55 rédies chez le premier, 77 rédies chez le second. Ces corps de taille très variable contiennent pour

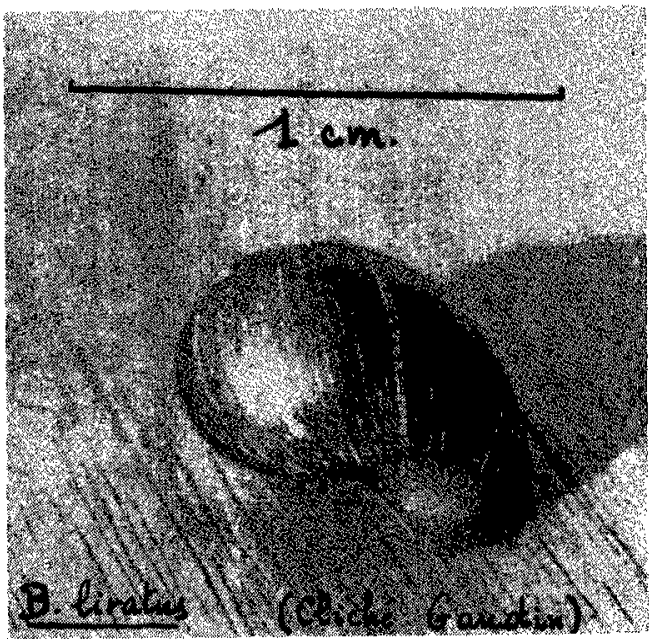

Fig. 13. - B. liratus. 
la plupart, de jeunes rédies sur le point de sortir par le tocostome. Par contre, à l'intérieur de 3 rédies de grande taille $(850 \mathrm{\mu})$, se trouvent des éléments qui, d'après leur forme, la présence de taches oculaires et celle d'une queue, peuvent être rapportés à de jeunes cercaires.

Aucune cercaire n'est trouvée dans les tissus périphériques du mollusquc.

Le $30^{\mathrm{e}}$ jour, à la dissection d'un $B$. liratus, nous trouvons dans son hépato-pancréas 57 rédies petites-filles, 32 rédies filles et 8 rédies mères en voie de dégénérescence. Dans les tissus soustégumentaires, nous mettons en évidence 33 jeunes cercaires dont les taches oculaires, petites et bien arrondies, nous indiquent quil s'agit d'éléments trc̀s jeunes.

Poursuivant notre expérience, nous avons la chance de pouvoir faire émettre par un B. mariei le $35^{\mathrm{e}}$ jour après son infestation $82 \mathrm{cer}$ caires mûres que nous faisons enkyster sur des fragments de graminées. $\left({ }^{*}\right)$

L'émission des cercaires se produit dans les 10 minutes qui suivent l'exposition à la lumière, elles nagent dans l'eau pendant 20 à 30 minutes et ne tardent pas à se fixer sur un support (fragment de graminées, ou plus simplement paroi du récipient où a lieu l'expérience). Une fois fixées elles perdent leur queue et s'enkystent. L'enkystement semble terminé au bout de 30 à 40 minutes. La métacercaire enkystée ressemble à un petit grain noir aplati de 250 à $300 \%$ de diamètre visible à l'œil nu et où l'on ne distingue aucune structure interne.

A la dissection de ce mollusque, nous trouvons une centaine de rédies dans l'hépato-pancréas et un nombre incalculable de cercaires en voie de maturation dans les tissus sous-tégumentaires.

Pour B. liratus, nous n'obtenons l'émission de cercaires que le $54^{\mathrm{e}}$ jour après l'infestation des mollusques par les miracidia.

Au cours de cette expérience, aucun des Anisus crassilabrum, L. hovarum, B. madagascariensis disséqués n'a été trouvé infesté.

Une telle différence dans les durées d'évolution de $P$. cervi chez $B$. mariei et $B$. liratus nous incite à refaire un essai d'infestation avec uniquement $B$. liratus.

Notre troisième essai d'infestation a été fait sur $32 \mathrm{~B}$. liratus, avec une durée d'infestation de 4 heures.

(*) Pour obtenir l'émission de cercaires chez le mollusque, nous avons travaillé à l'étuve à $29^{\circ}-32^{\circ}$, en éclairant le cristallisoir avec une forte lampe électrique de 200 watts.
Comme au cours de la deuxième expérience, les sporocystes n'apparaissent que vers le $13^{e}$ jour après l"infestation, alors que les rédies ne peuvent être mises en évidence que vers le $20^{\mathrm{e}}$ jour. Les jeunes cercaires ayant émigré vers les tissus sous-tégumentaires ne sont trouvées que vers le $30^{\mathrm{e}}$ jour, et les cercaires mûres ne sont émises qu'à partir du $50^{e}\left(^{*}\right)$.

\section{INFESTATION DE L'HOTE DÉFINITIF}

En ce qui concerne l'infestation du ruminant par les métacercaires de $P$. cervi, nous avons fait avaler à un veau 80 kystes fixés sur des fragments de graminées (cercaires émises par un $B$. mariei infesté expérimentalement).

A l'autopsie de cet animal 76 jours après son infestation, nous avons retrouvé dans le réseau et dans la panse, 69 formes immatures de $P$. cervi.

Cette expérience ne nous permet pas de donner des précisions sur la durée de l'évolution chez le ruminant, de la métacercaire à la forme adulte pondant des ceufs.

Cette partie du cycle de $P$. cervi mérite pourtant d'être étudiée en détail car les auteurs ne sont pas d'accord au sujet des migrations qu'accomplit cet helminthe au cours de son développement chez le ruminant.

Il serait intéressant de connaître la durée du séjour des formes immatures dans l'intestin avant leur migration vers les réservoirs gastriques. Nollier et Schmid en 1927 ont signalé cette localisation des formes jeunes de $P$. cervi chez le mouton, mais n'ont donné aucune précision au point de vue cycle du parasite chez son hôte définitif.

\section{FORMES LARVAIRES}

Nous ne donnerons aucun détail au point de vue structure des sporocyste, rédie et cercaire de $P$. cervi. Toutes ces formes ont été très bien étudiées et décrites par Loos en 1896 puis par Brumpt en 1929 et 1936. Nous nous contenterons de publier quelques microphotographies permettant de donner un aperçu des

(*) D'après les travaux de Loos en 18\%, l'évolution des formes larvaires de $P$. cervi chez Bulinus contortus et Bulinus forskali, serait en Egypte pour une température de $15^{\circ}$ à $20^{\circ}$, de 70 jours.

Au cours de ces infestations expérimentales, nous avons remarqué sur les $B$. liratus infestés par $P$. cervi ce que Brumpt avait déjà signalé en 1929 sur les $B$. contortus infestés par le même trématode en Corse, à savoir un retard dans la croissance des mollusques infestés.

Par contre, pour $B$. mariei, nous n'avons constaté aucune différence de croissance entre les spécimens infestés et ceux qui ne l'étaient pas. 


\section{SCHÉMA DU CYCLE EVOLUTIF DE "Paramphistomum cervi" A MADAGASCAR}

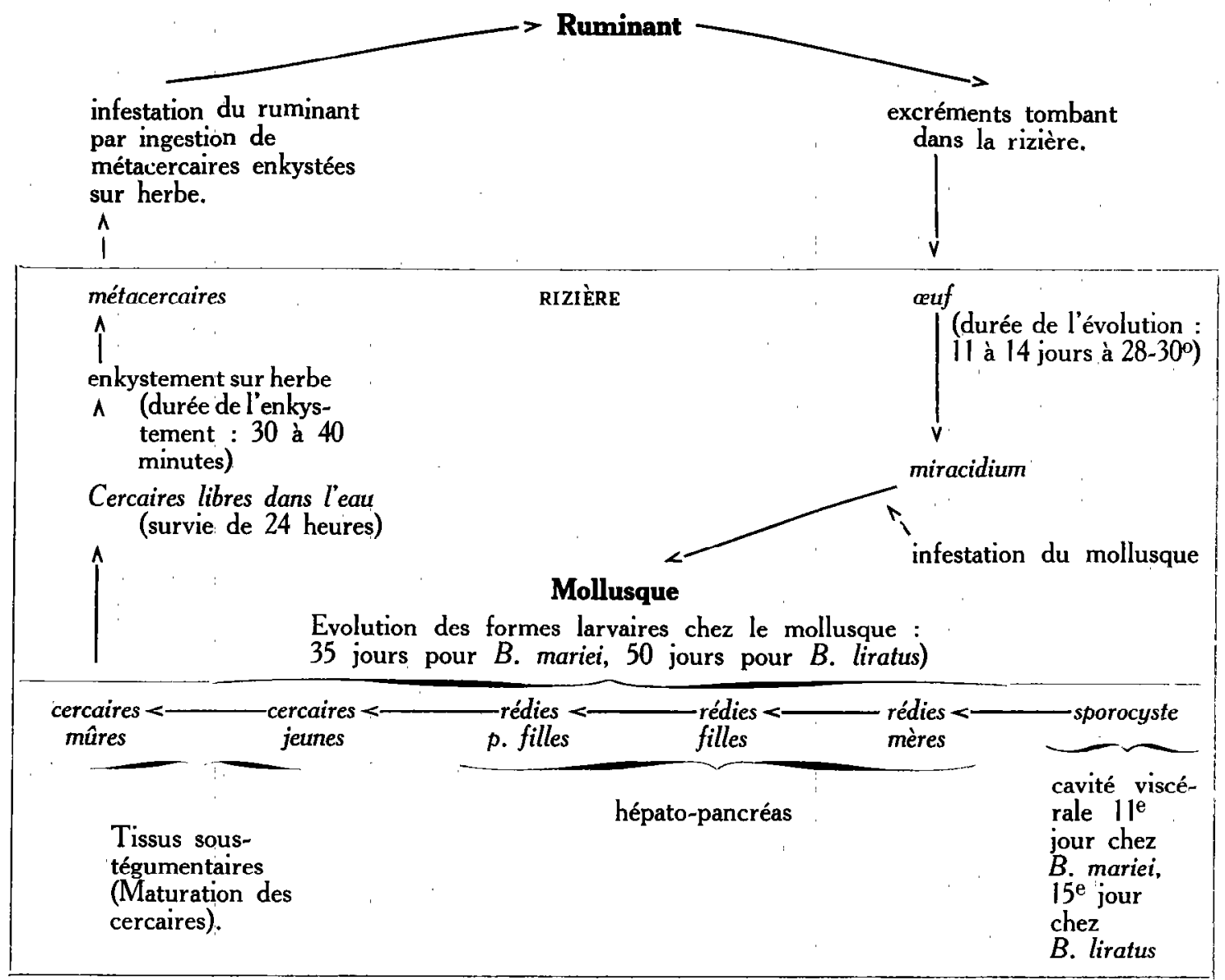

dimensions et de l'allure générale des stades larvaires trouvées chez le mollusque $\left(^{*}\right)$.

Nous tenons cependant à signaler, comme l'avaient déjà remarqué Filippi en 1855 , puis Loos en 1896, et enfin Brumpt en 1929, que chez les $\mathrm{Pa}$ ramphistomidae les cercaires quittent prématurément les rédies avant leur complète maturité. Elles émigrent vers les tissus périphériques du mollusque, et c'est seulement là, qu' elles acquièrent au bout d'un certain temps leurs caractères définitifs. Il y a en quelque sorte parasitisme de l'hôte intermédiaire par les cercaires libérées des rédies.

Au point de vue taxonomique, il est intéressant de noter que les sporocystes que nous avons

$\left(^{*}\right)$ Nous nous excusons de ne pas publier ici de microphotographies de la cercaire mûre de $P$. cervi. Les mouvements extrêmement rapides de ces éléments ne permettent pas de les photographier. Les essais de coloration vitale suivant la technique que nous avons utilisée pour le miracidium n'ont abouti qu'à des échecs. Les cercaires soumises à un tel traitement perdent immédiatement leur queue, et leur tête éclate dans les quelques secondes qui suivent la coloration. Nous n'avons pas obtenu de meilleurs résultats à l'aide du refroidissement. trouvés au cours de ces infestations expérimentales étaient en forme de sac oblong de 800 à $1.000 \%$ de long sur 300 à $350 \%$ de large, identiques à ceux qu'avaient décrits Loos en Egypte pour $P$. cervi. Contrairement à ce que prétend Nasmark (1937), Loos a bien décrit le cycle de $P$. cervi et non celui de $P$. microbothrium Fischoeder, 1901. En effet, si l'on examine la description et la figure que donne Dinnick en 1954 pour le sporocyste de $P$. microbothrium, il est facile de constater qu'il ne' ressemble en aucune manière à celui décrit par Loos.

Dinnick dit à ce sujet : "Fully developed sporocysts were up to $3.6 \mathrm{~mm}$ long, the elongated body being divided into parts, by a varying number of deep constrictions, each part containing embryo-balls and developing rediae". (*).

$\left(^{*}\right)$ "Les sporocystes bien développés mesuraient jusqu' à 3,6 mm. de longueur, leur corps allongé étant divisé en plusieurs sections par un nombre variable d'étranglements profonds, chaque section ainsi formée contenant des cellules embryonnaires et des rédies en voie de déve'oppement ". (traduction de la rédaction). 
Par ailleurs, les différences que nous signalons pour ces deux sporocystes nous permettent de dire, contrairement aux opinions de Nasmark (1937) et de Swart (1954), que $P$. cervi et $P$. microbothrium sont deux espèces différentes.

Au moment de terminer ce travail, nous prenons connaissance de l'excellente étude de Brygoo (1957) sur la répartition géographique des principales espèces de mollusques d'eau douce existant dans la Grande Ile.

Grâce à ce travail, beaucoup de problèmes d'épidémiologie pourront être résolus tant au point de vue bilharzioses humaines, qu'helminthiases des animaux domestiques.

\section{CONCLUSION}

L'infestation expérimentale de mollusques élevés au laboratoirc nous a permis de connaitre les hôtes intermédiaires de $P$. cervi à Madagascar. Ce sont : Bulinus mariei et Bulinus liratus.

Au point de vue durée d'évolution des formes larvaires chez ces hôtes intermédiaires à $28^{\circ}-30^{\circ}$, elle est beaucoup plus longue ( 50 jours environ) chez $B$. liratus que chez $B$. mariei ( 35 jours environ).

D'après les cartes de répartition des mollusques d'eau douce existant à Madagascar, $B$. mariei n'existe pas sur les hauts plateaux. On le trouve uniquement dans les régions Nord-Ouest, Nord, le littoral Est et les régions de basse altitude du Sud de l'Ile. C'est un mollusque vivant dans les rizières des contrées chaudes. (Brygoo, 1957).

Par contre $B$. liratus est pratiquement répandu dans tout Madagascar.

La réceptivité de ces deux espèces à l'infestation par $P$. cervi explique la dispersion de ce trématode dans toute l'lle.

Comme dans les autres parties du monde, ce sont les bulins qui, à Madagascar, scnt les hôtes intermédiaires de cet helminthe.

\section{Laboratoire central de l'Elevage. Service de parasitologie Tananarive.}

\section{BIBLIOGRAPHIE}

Brumpt (E.). - Particularités évolutives peu connues des cercaires d'Amphistomidés. Ann. Parasit, hum. comp., 1929, 7, 262-72.

BRUMPT (E.). - Contribution à l'étude de l'évolution des Paramphistomidés. Param phistomum cervi et cercaire de Planor bis exustus. Ann. Parasit. hum. comp., 1936, 14, 552-63.
Brygoo (E. R.). - Mollusques et bilharzioses humaines à Madagascar. Enquêtes épidé miologiques 1955-1957. Arch. Inst. Pasteur, Madagascar, 1957, 26, 43-112.

DinNick (J.A.). - An Intermediate Host of the Common Stomack Fluke, Paramphistomum cervi (Schrank), in Kenya. East Afric. agric. J., 1951, 16, 124-5.

Dinnick (J.A.) et Dinnick (N. N.). - The Life Cycle of Paramphistomum microbothrium Fischoeder, 1901 (Trematoda, Paramphistomidae). Parasit., 1954, 44, 285-99.

FIlIPPI ( $\mathrm{Ph}$. de). - Deuxième mémoire pour servir à l'histoire génétique des Tréma todes. Mém. Acad. Sci. Turin, 1855, 13-5.

Crétillat (S.). - Note préliminaire sur la gastrothylose des jeunes zébus à Madar gascar. Rev. Elev. Méd. vét. Pays trop., 1957, 10 (3), 221-30.

Grjebine (A.). - Note sur les mollusques de la région de Marovoay (Madagascar). Le Naturalistc Malgache, 1953, 5, 217-9.

Grjebine (A.) et MenaChe (M.). - Enquête mala cologique et hydrobiologique sur les mol lusques vecteurs de bilharziose dans le district d'Ambositra. Mém. Inst. Sci. Madagascar, 1953, série A, 8, 87-110.

Loos (A.). - Recherches sur la faune parasitaire de l'Egypte. Mém. Inst. égyptien, 1896, 3, 1-252.

NASMARK (K. E.). - A Revision of the Trema tode Family Paramphistomidae. (Inaug. Dissert. Uppsala. Zoologisk.) Bidrag. Fran. Uppsala., 1937, 16, $301-566$.

Neumann (G.). - Traité des maladies parasitaires non microbiennes des animaux domestiques. $2 \mathrm{e}$ édition, Paris, 1892.

Noller (W.) et Schmid (F.). - Zur Kenntnis der Entwingsklung von Paramphisto. mum cervi (Schrank) s. Amphistomum conicum Zeder. Sitz. Ber. Ges. Naturf. Fr., Berlin, 1927, p. 148.

Sonsino (P.). - Studi sui parassiti di molluschi di aqua dolce dintorni di Cairo in Egitto. Leuckart's Festchrift, 1892, 134-47.

Swart (P. J.). - The Identity of so Called Pa ramphistomum cervi and $P$. explanatum, two Common Species of Ruminant Trematodes in South Africa. Onderst. $J$. vet. Res., 1954, 26 (3), 463 73.

TAKAHASI (S.). - Ueber die Entwickelungsgeschi chte des Paramphistomum cervi. Anal. in Centralblatt f.d.ges. Hyg., Berlin, 1928, 18, 278.

Timon-David (J.). - Cycle évolutif d'un trématode Cyclocoelidé Pseudhyptiasmus dollfusi T.D., 1950. Recherches expérimentales. Ann. Parasit. hum. comp., 1955, 30, 43-61. 


\section{SUMMARY}

\section{Contribution to the determination of intermediate hosts and the study of life cycle of Paramphistomum cervi (Schrank, 1790), (Trematoda, Paramphistomidæ), in Madagascar.}

Up to now, no determination had been made of the life cycle of any trematode in Madagascar. Various larval forms believed to be of the Paramphistomidae had been found in fresh-water snails. The experimental life cycle of $P$. cervi is now described. Of five species of snails reared in the laboratory and placed in contact with the miracidia of $P$. cervi, only two species Bulinus mariei and $B$. liratus became infested. Mature cercaria emerged in 35 and 50 days approximately from these species respectively, after infestation, at a temperature of $28-30^{\circ} \mathrm{C}$. $P$. cervi were recovered from the first and second stomachs of a ruminant which had been fed with grass to which the cysts of cercaria had become fixed. These cercaria had emerged from $B$. mariei experimentally infested. The susceptibility to infestation with $P$. cervi from $B$. mariei which is widespread over Madagascar, explains the extensive geographical distribution of this trematode over the island.

\section{RESUMEN}

\section{Contribución al conocimiento de los huéspedes intermediarios y al estudio del ciclo evolutivo de Paramphistomum cervi (Schrank, 1790), (Trematoda, Paramphistomidæ), en Madagascar.}

En Madagascar, hasta entonces, ningùn ciclo de tremátode habia sido descrito o determinado; en los moluscos de agua dulce disecados, diferentes formas larvarias pudiendo ser identificadas como Paramphistumidae habian sido reconocidas. El autor ha realizado en su laboratorio el ciclo experimental de Paramphistomum cervi. De cinco especies de moluscos criados en el laboratorio y puestas en presencia de miracidias de $P$. cervi, sólo dos especies, Bulinus mariei y $B$. liratus, se han infestado: Ha podido obtener la expulsión de cercarias maduras por $B$. mariei y por $B$. liratus alrededor de 35 y 50 dias respectivamente, despues de la infestación, a una temperatura de $28-30^{\circ} \mathrm{C}$. Ha encontrado $P$. cervi en la redecilla y la panza de un rumiante al cual habia hecho ingerir quistes fijados a gramineas, procedentes de cercarias emitidas por un $B$. mariei infestado experimentalmente.

La receptividad a la infestación por $P$. cervi de $B$. mariei que se encuentra en los regiones bajas de Madagascar, y de B. liratus, que está repartido en todo Madagascar, explica la dispersión de este tremátode en toda la isla. 